\title{
Isolated rupture of the superficial vein of the penis
}

\author{
Alper Eken, MD, FEBU; $;^{*}$ Meltem Acil, MD; ${ }^{\dagger}$ Taner Arpaci, $M D^{\varsigma}$
}

*Acibadem Adana Hastanesi Urology Department; Acibadem University Vocational School of Health, Adana, Turkey; ${ }^{\star}$ Acibadem University Department of Anesthesiology and Reanimation, Istanbul, Turkey; §Acibadem University Department of Radiology and Imaging, Istanbul, Turkey

Cite as: Can Urol Assoc J 2014;8(5-6):e371-3. http://dx.doi.org/10.5489/cuaj.1712 Published online May 21, 2014.

\section{Abstract}

Penile emergencies are rare but when they do occur, prompt diagnosis and treatment are warranted. Emergent conditions of the male genitalia are mainly traumatic, vascular or infectious. Penile emergencies are usually caused by trauma to the penis, during sexual intercourse or manipulation of an erect penis during masturbation. One of the traumatic vascular penile emergencies is superficial penile dorsal vein rupture. This is a rare condition, with just a few reported cases. It is usually taken into differential diagnosis with the other acute penile injuries that present, such as acute penile edema or ecchymosis. We report a case of 59-year-old male with a superficial penile dorsal vein rupture which occurred during manipulation of the erect penis.

\section{Introduction}

Acute onset of swelling, ecchymosis and pain of the penis during sexual intercourse has been known to suggest penile fracture, unless proven otherwise. Predisposing factors are mostly vigorous sexual intercourse or forceful manipulation of the erected penis. ${ }^{1}$ Nonetheless, there are a few cases of injuries to the dorsal vein or dorsal artery mimicking penile fracture. Superficial dorsal vein injury or "false fracture" should be taken into differential diagnosis along with penile fracture. Contrary to the situation in penile fracture, patients with superficial dorsal vein injury do not present with a "click" or "snap" sound. ${ }^{2}$

\section{Case Report}

A 59-year-old male was admitted to the emergency clinic with ecchymosis of the penis. Painless ecchymosis had developed during the manipulation of the erected penis 3 hours before. He denied hearing a "snap" sound, which is usually followed by penile detumescence or pain. His physical examination revealed ecchymosis of the base of the circumcised penis with normal appearance of the glans (Fig. 1). Tunical rupture was not detected on physical examination. Penile ultrasonography was carried out and revealed only non-specific hematoma at the base of the penis.

Penile exploration was performed under spinal anesthesia via degloving incision. The tunica albuginea and the penile urethra were examined for injuries and both were intact. The only pathological finding was superficial penile dorsal vein rupture at the base of the penis. Ligation was performed at the base and the operation was ended. Penile hematoma was reduced in a few days and recovery from ecchymosis was observed in 2 weeks. Six months after the operation, he reported improved erectile function without difficulty in intercourse (Table 1).

\section{Discussion}

Superficial penile dorsal vein rupture can be caused by blunt trauma to an erect penis, particularly when initiating or during sexual intercourse. The blunt trauma causes venous tears and lacerations, which bleed and form a hematoma. Even though there are no large cohorts to provide data on dorsal penile vein rupture and the associated risk factors, BarYosef and colleagues have described their experience with 9 patients. ${ }^{3}$ They suggested that circumcision could be one of the risk factors for dorsal vein rupture due to stretching of the penile skin during intercourse. Similarly, Baran and colleagues concluded that circumcision could have been the mechanism of injury in their patient with superficial penile dorsal vein rupture after surgical exploration. ${ }^{4}$

Patients with rupture of the superficial dorsal vein of the penis may show symptoms which mimic a penile fracture. However, penile fracture has a quite distinct clinical presentation. Most patients with penile fracture would report hearing a "snap" sound, which is followed by pain, penile detumescence, and late appearing swelling, hematoma and penile deformity. ${ }^{3,4}$ When there is associated urethral injury, we sometimes notice concurrent urethral bleeding, difficulty 


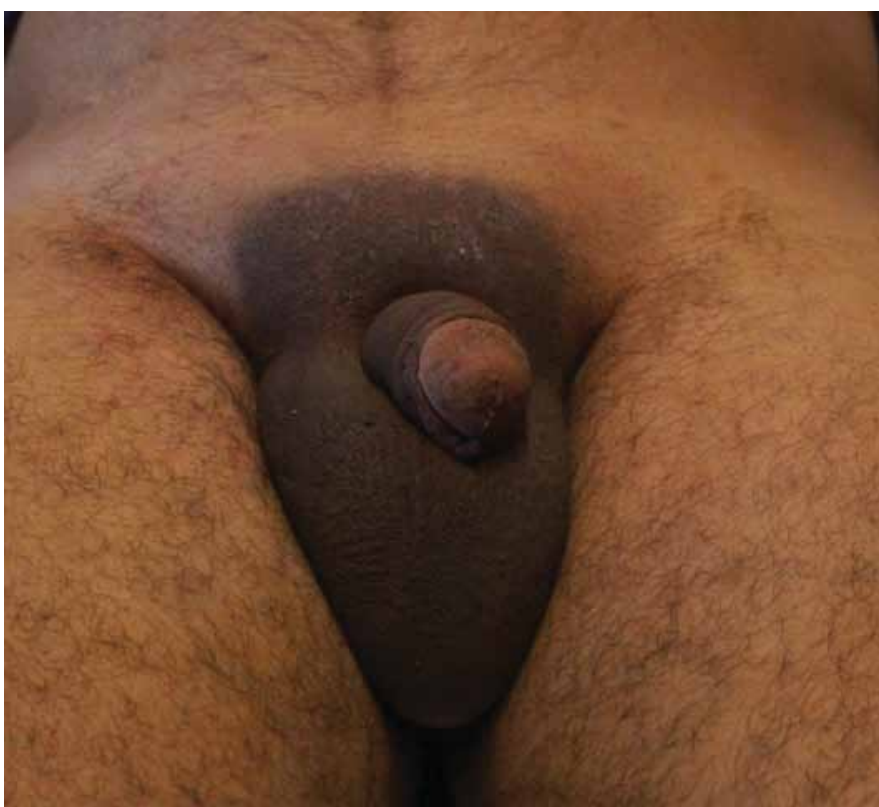

Fig. 1. Ecchymosis of the penis base of a circumcised penis with normal appearance of the glans.

voiding and hematuria. ${ }^{5}$ However, patients with rupture of the superficial dorsal vein of the penis may not describe the classic "snap-pop" or rapid detumescence. ${ }^{4}$ Patients with superficial penile dorsal vein rupture can experience bleeding from the ruptured vein and can present with a dorsally-swollen and mildly painful penis, which usually occurs within 24 hours following sexual intercourse. The dorsal hematoma occurs spontaneously and is not accompanied by immediate detumescence. The dorsally-swollen penis will reveal an ecchymosed area at the base. Nonetheless, the glans usually appears normal. ${ }^{4}$ In the present case, neither a "snap" sound nor rapid detumescence was reported by the patient. On physical examination, we found a hematoma at the base with normal appearance of the glans.

Ultrasound has a limited role and low sensitivity in the diagnosis of superficial penile dorsal vein rupture. Vasculature of the penis, including superficial dorsal vein, are well-evaluated with Doppler ultrasound. It can help to diagnose the discontinuity or tear of the dorsal vein and exclude the rupture of the cavernosal bodies. It is wellaccepted that Doppler sonography is the best and fastest radiological method to diagnose the superficial dorsal vein rupture. It can also allow us to follow-up patients to visualize the resolution of the ecchymosis and the normal penile blood flow after treatment. Magnetic resonance imaging (MRI) has also been used to visualize the superficial dorsal vein. It may detect rupture of the superficial dorsal vein. However, MRI and cavernosography are mostly recommended to depict discontinuity of the tunica albuginea and diagnose the penile fracture. ${ }^{6-8}$ The precise diagnosis can be confirmed only after surgical exploration. ${ }^{5,9}$ The few reported cases of rupture of the superficial dorsal vein of the penis sometimes imitate a penile fracture; this rupture is thus sometimes called a "false fracture." In such circumstances, confirmatory diagnosis can be achieved through surgical exploration to exclude penile fracture. The precise role of diagnostic investigations has not been established. ${ }^{4}$ If signs of cavernosal tearing are lacking during surgical exploration of patients with acute penile injury, then this could suggest that there is an unidentified vascular trauma, and the precise diagnosis ought to be rupture of the superficial or deep dorsal vessels or their branches. Consequently, these conditions may be renamed as vascular injuries of the penis, which may be subdivided further into superficial or deep, based on the hematoma location (i.e., either beneath or over the Buck's fascia). Superficial penile dorsal vein rupture affects the dorsal veins over the Buck's fascia., ${ }^{4,9,10}$

The management of the superficial penile dorsal vein rupture can either be conservative or surgical. ${ }^{4,10}$ Conservative management entails the use of anti-inflammatory drugs, as well as compressive bandage. Conservative treatment results in complete recovery without sequelae. Although conservative treatment is sometimes adequate to manage dorsal vein rupture, surgical exploration and/or intervention may be required for the differential diagnosis. Surgical exploration and intervention also allow ligation of the ruptured vessel and can reveal concomitant injuries or prevent further hematoma formation. ${ }^{4,9}$ Surgical exploration also provides good functional and cosmetic postoperative results, and is indicated when there is reasonable doubt regarding the pre-

\section{Table 1. Comparison preoperative (4 months prior to dorsal vein rupture) and 6-month postoperative IIEF-5 scores}

hard enough for penetration?

During sexual intercourse, how often were you able to maintain your erection after you had penetrated (entered) your partner?

During sexual intercourse how difficult was it to maintain your erection to the completion of intercourse?

When you attempted sexual intercourse, how often was it satisfactory for you?
3

3

$\begin{array}{cc}\text { Preoperative score } & \text { 6-month postoperative score } \\ 3 & 4 \\ 3 & 4 \\ 2 & 4 \\ 4 & 2 \\ 2 & 5\end{array}$

14: mild to moderate 19: mild 
cise diagnosis. ${ }^{10}$ Both management methods were discussed with the patient in the present case. Surgical intervention was carried out at the patient's request.

The prognosis after surgery is excellent, albeit some researchers maintain that long-term success depends on careful patient selection. ${ }^{11}$ In general, Sharma ${ }^{12}$ described a case where a high index of clinical suspicion resulted in the diagnosis of superficial penile dorsal vein rupture without surgical exploration. In this case, the patient recovered successfully with conservative management. Gil and colleagues ${ }^{13}$ presented a male patient with a 24 -hour evolution painless hematoma following sexual intercourse. Based on its evolution and the physical characteristics, the authors adopted a conservative approach, which resulted in complete recovery without sequels.

\section{Conclusion}

In our case, the prognosis was excellent. The patient experienced improved erectile function (Table 1).

Competing interests: Dr. Eken, Dr. Acil and Dr. Arpaci all declare no competing financial or personal interests.

This paper has been peer-reviewed.

\section{References}

1. Agarwal MM, Singh SK, Sharma DK, et al. Fracture of the penis: A radiological or clinical diagnosis? A case series and literature review. Can I Urol 2009;16:4568-75.

2. Perlmutter AE, Roberts L, Farivar-Mohseni $H$, et al. Ruptured superficial dorsal vein of the penis masquerading as a penile fracture: Case report. Can I Urol 2007;14:3651-2.

3. Bar-Yosef $Y$, Greenstein A, Beri A, et al. Dorsal vein injuries observed during penile exploration for suspected penile fracture. J Sex Med 2007;4:1142-6. http://dx.doi.org/10.1111/i.1743-6109.2006.00347.x

4. Baran C, Topsakal M, Kavukcu E, et al. Superficial dorsal vein rupture imitating penile fracture. Korean J Urol 2011;52:293-4. hitp://dx.doi.org/10.4111/kju.2011.52.4.293

5. El-Assmy A, El-Tholoth HS, Abou-El-Ghar ME, et al. False penile fracture: Value of different diagnostic approaches and long-term outcome of conservative and surgical management. Urology 2010;75:1353-6. http://dx.doi.org/10.1016/i.urology.2009.11.086

6. Kirkham A. MRI of the penis. Br J Radiol 2012;85(Spec Iss 1):86-93.

7. Avery LL, Scheinfeld MH. Imaging of penile and scrotal emergencies. Radiographics 2013;33:721-40. http://dx.doi.org/10.1148/rg.333125158

8. Koifman L, Cavalcanti A, Manes $\mathrm{CH}$, et al. Penile fracture-experience in 56 cases. Int Braz J Urol 2003;29:35-9. http://dx.doi.org/10.1590/S1677-55382003000100007

9. Simsek A, Kurt 0, Verim LN, et al. Rupture of superficial dorsal vein of penis. Arch Ital Urol Androl 2011;83:102-4.

10. Punal Pereira A, Perez Fentes D, Touce Caamano V, et al. Rupture of the superficial vein of the penis: Therapeutic options. Arch Esp Urol 2010;63:871-3.

11. Schultheiss $D$, Truss $M C$, Becker AJ, et al. Dorsal ligation of the penile vein-prognostic factors and long-term outcome. Urologe A 1997;36:351-5.

12. Sharma GR. Rupture of the superficial dorsal vein of the penis. Int I Urol 2005;12:1071-3. http:// dx.doi.org/10.1111/i.1442-2042.2005.01212.x

13. Arce Gil J, Rodriguez-Ledesma JM, Mavrich HV. Rupture of the superficial dorsal vein of the penis. Actas Urol Esp 2006;30:215-7. http://dx.doi.org/10.1016/S0210-4806(06)73425-8

Correspondence: Dr. Alper Eken, Acibadem Adana Hastanesi Urology Department, Cumhuriyet Cad № 66, Postal Code: 01130 Adana, Turkey; alpereken@yahoo.com 